\title{
Lows of Wear Process of the Friction Pair "0.45\% Carbon Steel-Polytetrafluoroethylene" during Sliding from the Position of Fracture Mechanics
}

\author{
Aleksandr V. Bogdanovich \\ Department of Theoretical and Applied Mechanics, Belarusian State University, Minsk, Belarus \\ Email: bogal@tut.by
}

How to cite this paper: Bogdanovich, A.V. (2019) Lows of Wear Process of the Friction Pair " $0.45 \%$ Carbon Steel-Polytetrafluoroethylene" during Sliding from the Position of Fracture Mechanics. World Journal of Mechanics, 9, 95-104.

https://doi.org/10.4236/wjm.2019.95007

Received: March 19, 2019

Accepted: May 2, 2019

Published: May 5, 2019

Copyright (c) 2019 by author(s) and Scientific Research Publishing Inc. This work is licensed under the Creative Commons Attribution International License (CC BY 4.0).

http://creativecommons.org/licenses/by/4.0/

\begin{abstract}
The results of the tests for a friction pair "a cylindrical specimen made of $0.45 \%$ carbon steel-a counter specimen-liner made of polytetrafluoroethyleneF4-B" during sliding friction are presented. The test results at different levels of contact load are analyzed using the Archard's equation and are presented as a friction fatigue curve. The concept of the frictional stress intensity factor during sliding friction is introduced, and an expression that relates the wear rate to this factor and is close in shape to the Paris equation in fracture mechanics is proposed.
\end{abstract}

\section{Keywords}

Sliding Friction, Contact Pressure, Wear, Frictional Stress Intensity Factor, Polymer

\section{Introduction}

Among various theories of mechanical wear of solids in recent decades, fatigue theory has been widely recognized [1] [2] [3]. It turns out to be true if the contact load is relatively small, and the deformation of the friction surface is predominantly elastic.

One characteristic of this type of wear is the material damage under the repetitive action of compressive, tensile and shear deformations during cyclic loading caused by the interaction of the polymer with the hard and blunt projections on the rough surface during sliding, which gives rise to the generation and development of cracks, and which can be assisted by the presence of defects [4]. Some authors modify the term fatigue wear to frictional or rolling wear if the polymer presents a low tearing strength and slides on smooth counterfaces with high fric- 
tion coefficient, causing roll formation at the sliding interface and tearing of the rolled fragment [5].

According to several studies, the interaction of the abrasive particles with the polymer produces deformation and tensile, compressive and shear stresses in the worn surface layer, forming in it fatigue cracks due to the repetitive action of these interactions [6]. Other investigations indicate that the largest shear stress takes place at a certain depth under the surface, this point being nearer to the surface as the friction force increases [7] [8]. On the other hand, the deformation of the material is greatest at the surface, which is propitious to the formation of cracks, but at the same time the compressive stress is also at its greatest in this area and restrains crack formation. With the increase of distance to the worn surface, the compressive stress decays faster than the strain, so that at some depth in the worn surface layer, the stress is almost pure shear stress and cracks are able to form more easily [9].

As known within the models based on contact mechanics, a model of particular relevance and broadly used is that proposed by Archard [10] [11], which is commonly expressed as:

$$
W=\frac{k}{H} F_{N} \gamma,
$$

where $W\left[\mathrm{~mm}^{3}\right]$ is the worn volume, $F_{N}[\mathrm{~N}]$ the applied normal load, $\gamma[\mathrm{m}]$ the sliding distance, $k$ the non-dimensional wear coefficient particular to the contact pair characteristics and $H\left[\mathrm{~N} / \mathrm{mm}^{2}\right]$ the material hardness. When interpreting experimental situations, the hardness of the uppermost layer of material in the contact may not be known with any certainty and consequently a rather more useful quantity than the value of $k$ alone is the ratio $k / H\left[\mathrm{~mm}^{3} \cdot \mathrm{N}^{-1} \cdot \mathrm{m}^{-1}\right]$, named hereinafter as $K$ and which is known as the dimensional wear coefficient or specific wear rate [9].

According to Figure 1, three different stages are accepted for describing a typical wear process: a first running-in stage in which the wear uniformity in the contact pair is being set up by elimination of the micro-asperities of the surfaces, a second stationary stage where a constant wear rate has been attained and the surface or surfaces are worn in a steady and uniform way, and a third accelerated stage where the wear rate increases in an exponential way and leads to catastrophic failure.

Archard's law referred to in Equation (1) is usually applied to the stationary stage. With the rest of the variables of the equation well known and without variation, the constant $K$ can be considered as the characteristic wear coefficient of the wear process under study.

In this paper, the kinetic process of wear of the steel-polymer mechanical system is analyzed using fatigue fracture mechanics approaches.

\section{Sliding Friction Tests and Their Results}

Tests on sliding friction of the metal-polymer friction pair were carried out ac- 
cording to the shaft-liner scheme. The sample-shaft 1 made of $0.45 \%$ carbon steel with $10 \mathrm{~mm}$ diameter of working part was cantilevered in the spindle 2 of the upgraded testing machine UKI-6000-2 and rotated at a frequency of 3000 $\mathrm{min}^{-1}$ (Figure 2). The counter specimen-liner 3 which is a $10 \times 10 \times 10 \mathrm{~mm}$ cube made of polymer polytetrafluoroethylene (PTFE) F4-B was pressed to the dangerous section of the specimen 1 with a contact load $F_{N}$, the value of which was set using a special tool and kept constant during the test of each pair of specimen-liner.

In the process of testing, a drip supply of a lubricant-Universal All-Seasonal Engine Oil "Lukoil Super 15W-40"-was provided and the measurement the linear wear of the friction pair using an indicator head with an accuracy of $2 \mu \mathrm{m}$ was performed. Since the steel sample in the test pair did not wear out, all wear was obtained by a polymer liner. The liner wear equal to $i_{\text {lim }}=1000 \mu \mathrm{m}$ was taken as the limit state.

The test results of the friction pair with the contact load $F_{N}$ equal to 150,180 , 280,350 and $450 \mathrm{~N}$ are shown in the form of kinetic graphs of the dependence of wear $i[\mu \mathrm{m}]$ on the number $N$ of rotates [cycles] in Figure 3 . These graphs correspond to the stationary stage of the wear process. Therefore, the Archard's equation can be applied to the above test results.

On the other hand, based on the fatigue theory of mechanical wear, the results of the tests can be represented as fatigue (Weller) curves in the coordinates of the contact load $F_{N}$ the number $N$ of cycles before the limit state (for $i_{\text {lim }}=1000$ $\mu \mathrm{m}$ ) of the polymer liner (Figure 4). As can be seen from Figure 4, the fatigue

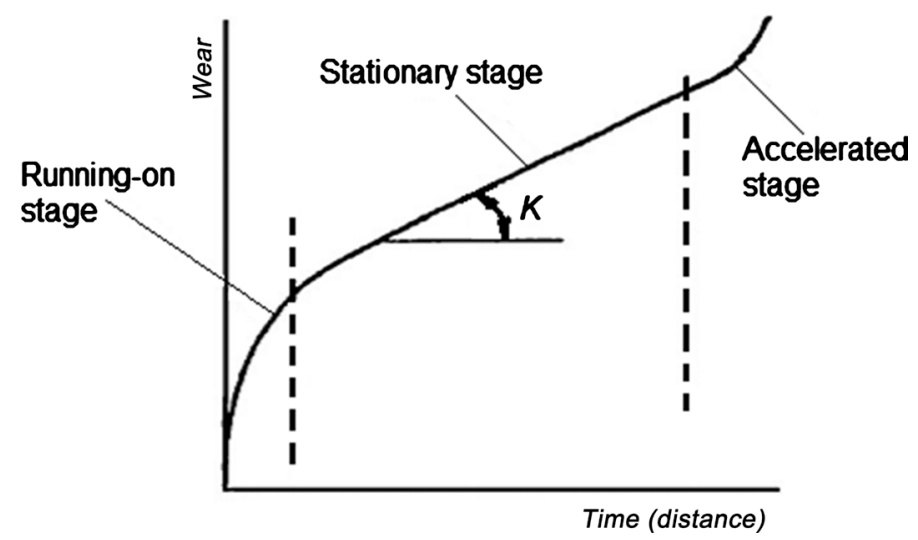

Figure 1.Typical wear curve in a tribological system [2].

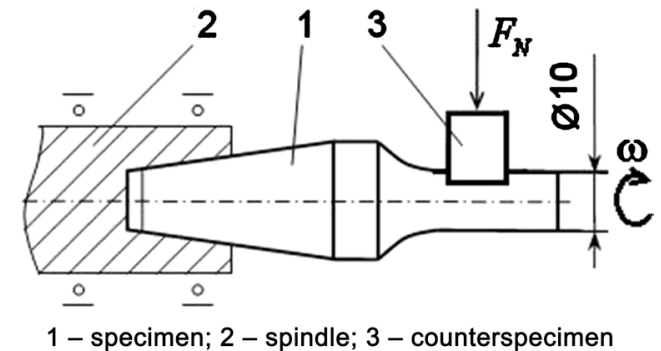

Figure 2. Sliding friction test scheme. 


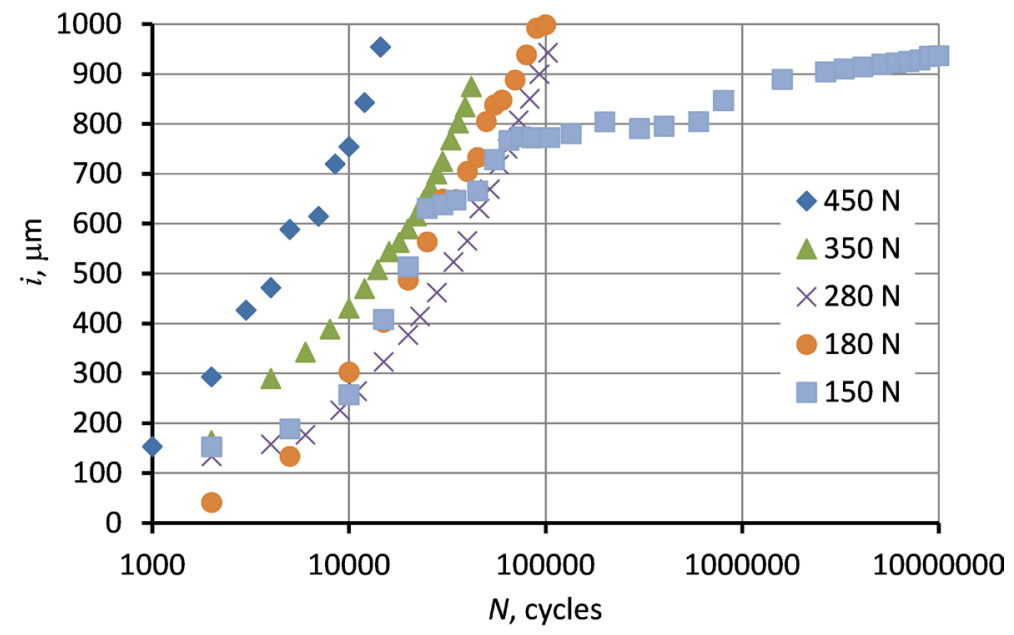

Figure 3. Kinetic curves of wear of the polymer liner during the sliding friction test with contact load 450, 350, 280, 180 and $150 \mathrm{~N}$.

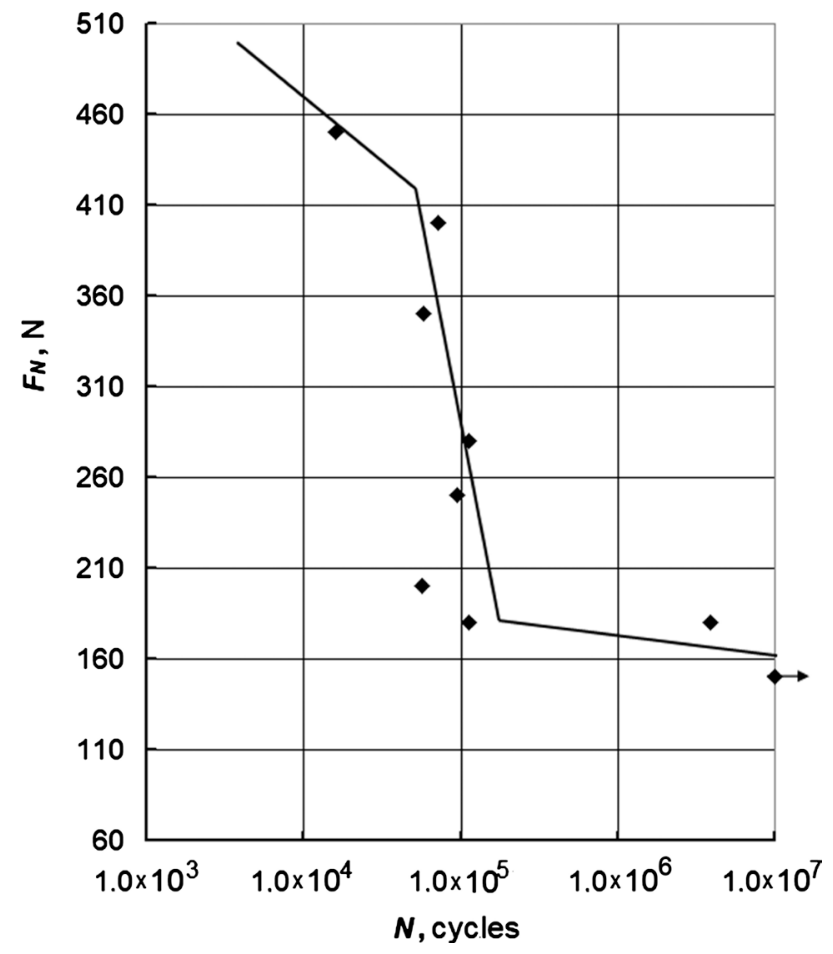

Figure 4. Friction fatigue curve of a polymer liner.

curve consists of three branches: the left branch with a slope (this is a region of quasi-static fracture to approximately $N=7.2 \times 10^{4}$ cycles, $F_{N}=400-450 \mathrm{~N}$ ), an average line located almost vertically (this is the area of low-cycle destruction $N$ $=7.2 \times 10^{4}-9 \times 10^{4}$ cycles, $\left.F_{N}=165-400 \mathrm{~N}\right)$, and the right one with a large slope (this is the area of multi-cycle destruction $N>1 \times 10^{5}$ cycles, $F_{N}<165 \mathrm{~N}$ ).

Let's try to describe the test results using the Archard's equation. In this case, in Equation (1) we write linear wear $i$ instead of volume wear $W$, since they are proportional to each other. The sliding distance $\gamma$ is replaced by the number $N$ of loading cycles (these quantities are also proportional to each other). In Figure 5 

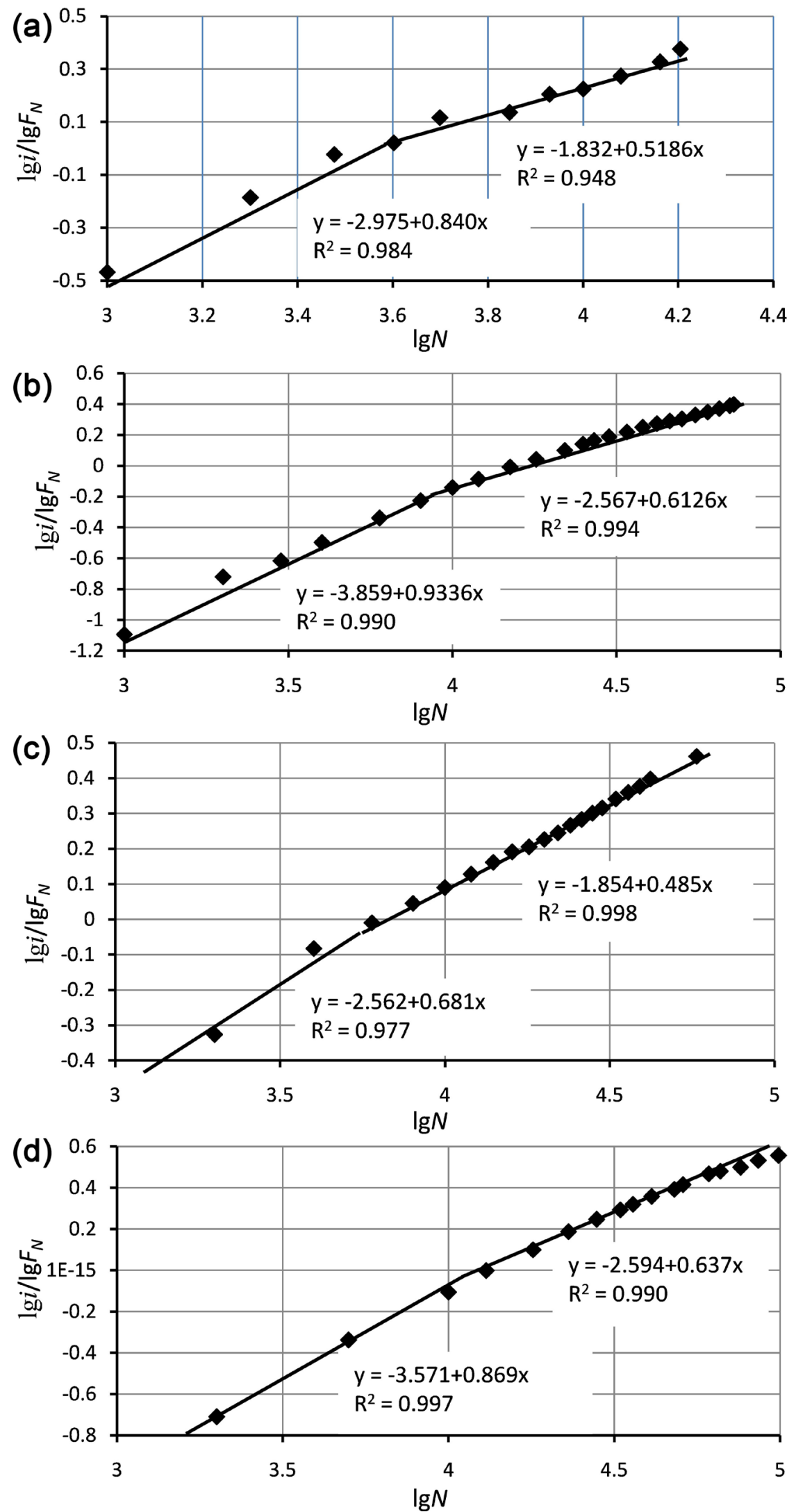

Figure 5. Graphs of the relationship of the ratio $i / F_{N}$ on the number $N$ of loading cycles of the polymer liner under contact load 450 (a), 400 (b), 350 (c) and 250 (d) N. 
shows graphs of the ratio il $F_{N}$ against the number $N$ of loading cycles, plotted in logarithmic coordinates according to the test results of the friction pair under study.

As can be seen from Figure 5 the experimental points can be quite satisfactorily described by a linear equation of the form $y=a x+b$. At the same time, in the studied range of $i / F_{N}$ versus $N$, each graph can be represented as consisting of two linear dependencies, the values of the parameters $a$ and $b$ of the equations of which are shown in Figure 5. Apparently, the left part of the dependences in Figure 5 corresponds to the stage of steady wear, and the right-hand side to the stage of accelerated wear in accordance with the typical wear curve in Figure 1.

Analysis of graphs in Figure 5 shows that using the Archard's equation it is not possible to describe all the test results of the material under study at different values of the contact load.

\section{Wear Process of Polymer from the Position of Fracture Mechanics}

Some authors, such as Martinez et al. [9], Thomas et al. [12], Cho and Lee [13], have carried out investigations into polymers relating the mechanism of wear by abrasion and the mechanical fatigue process of crack growth theories. For the same material, they have observed that within the ranges of stable crack growth rate in fatigue and uniform debris detachment in wear, the slope of the abrasion rate in the wear process is similar to that of the crack growth rate in the fatigue mechanism, suggesting that both phenomena are related, the abrasion of the material occurring as a result of repeated crack propagation on a small scale.

Regarding the fatigue crack process, Figure 6 shows the different zones in

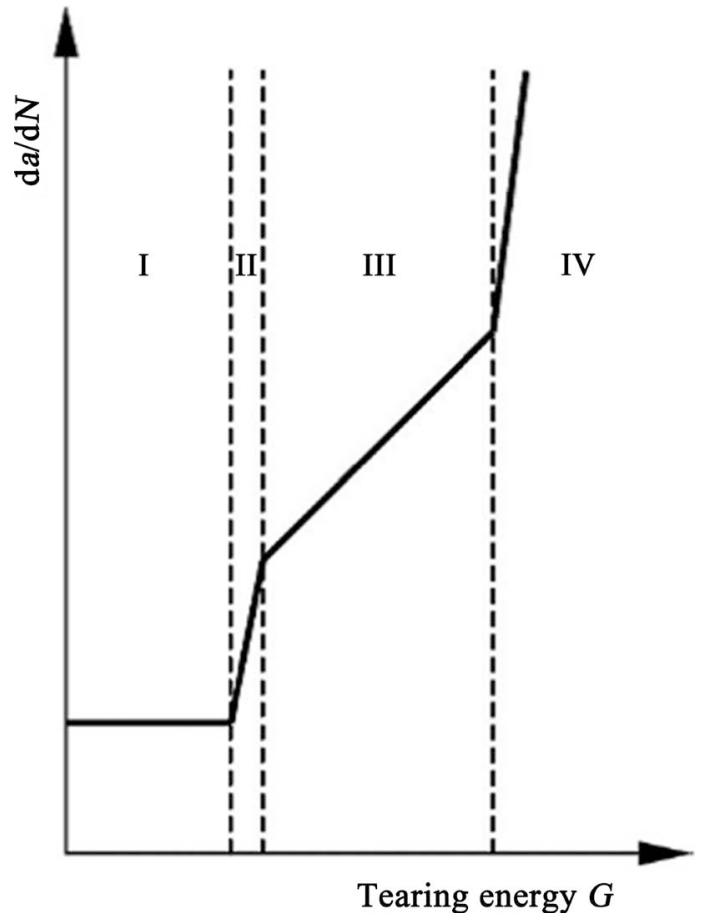

Figure 6. Crack growth characteristics for polymer [9]. 
which the fatigue crack growth behavior is divided for a polymer [9] [14]. This is known as the crack growth characteristic and is divided into four regions. In region I, the strain energy release rate or tearing energy $G$, defined as the partial derivative of the total elastic strain energy stored in an article containing a crack by the area of one fracture surface of the crack, is less than the threshold tear energy $G_{0}$, hence no mechanical crack growth occurs. In region II, the region of slow crack growth, the crack growth is dependent on both ozone and mechanical factors in an additive way. In region III, a power law dependency between the crack growth rate and the tearing energy is found as follows:

$$
\frac{\mathrm{d} a}{\mathrm{~d} N}=B G^{\beta},
$$

where $a[\mathrm{~mm}]$ is the crack length, $N$ is the number of cycles, $B$ and $\beta$ are material constants. Depending on the polymer type, the value of $\beta$ lies between 1.5 and 6; in this region, stable crack growth takes place. Region IV corresponds to a rapid and unstable crack growth and therefore to the region of catastrophic failure.

In addition to the energy $G$, the stress intensity factor proportional to the value of $G$ is often used as a control parameter for crack growth in fracture mechanics. As known, for a sample with limited dimensions the crack growth under the action of shear stresses $\tau$ is controlled by the shear stress intensity factor

$$
K_{\tau}=Y \tau \sqrt{\pi a}
$$

where $Y$ is the correction function that takes into account the geometry of the sample and its loading circuit.

In the case of volumetric damage during mechanical fatigue the crack size $a$ characterizes the degree of material damage, while the surface damage caused by sliding friction is characterized by the value $i$ of wear. Instead of tangential shear stress $\tau$ under friction, we can apply the so-called specific friction force or friction stress $\tau_{w}$ equal to [3]

$$
\tau_{w}=f p_{a}=f \frac{F_{N}}{A_{a}},
$$

where $f$ is the friction coefficient; $p_{a}$ is the average contact pressure; $A_{a}$ is the nominal contact area. Consequently, with reference to sliding friction, taking into account the assumptions made and (4), expression (3) can be written as

$$
K_{\tau_{w}} \sim \tau_{w} \sqrt{i} \sim p_{a} \sqrt{i} \sim \frac{F_{N}}{A_{a}} \sqrt{i} .
$$

Thus, using expression (5), it is possible to estimate the frictional stresses intensity factor under sliding friction. Obviously, the damage rate $\Delta a / \Delta N$ with the growth of fatigue cracks can be matched to the wear rate $\Delta i / \Delta N$ (in discrete form). Then for the wear rate during sliding friction, we obtain an expression close in form to (2):

$$
\frac{\Delta i}{\Delta N}=B^{*} K_{\tau_{w}}^{\beta^{*}} \sim B^{*}\left(\frac{F_{N}}{A_{a}} \sqrt{i}\right)^{\beta^{*}},
$$


where $B^{*}$ and $\beta^{*}$ are material constants characterizing the steady-state stage of the wear process.

The analysis of experimental data on the expression (6) showed their satisfactory compliance. In Figure 7 as an example the graph $\lg (\Delta i / \Delta N)-\lg K_{\tau W}$ for the contact load $280 \mathrm{~N}$ is plotted.

The generalized graph $\lg (\Delta i / \Delta N)-\lg K_{\tau w}$ for the test results for all levels of contact load is presented in Figure 8. It completely corresponds to the classical $S$-shaped curve of the dependence of the fatigue crack growth rate on the stress intensity factor known in fracture mechanics. If we compare the obtained graph with a typical dependence of the crack growth rate on tearing energy for polymers

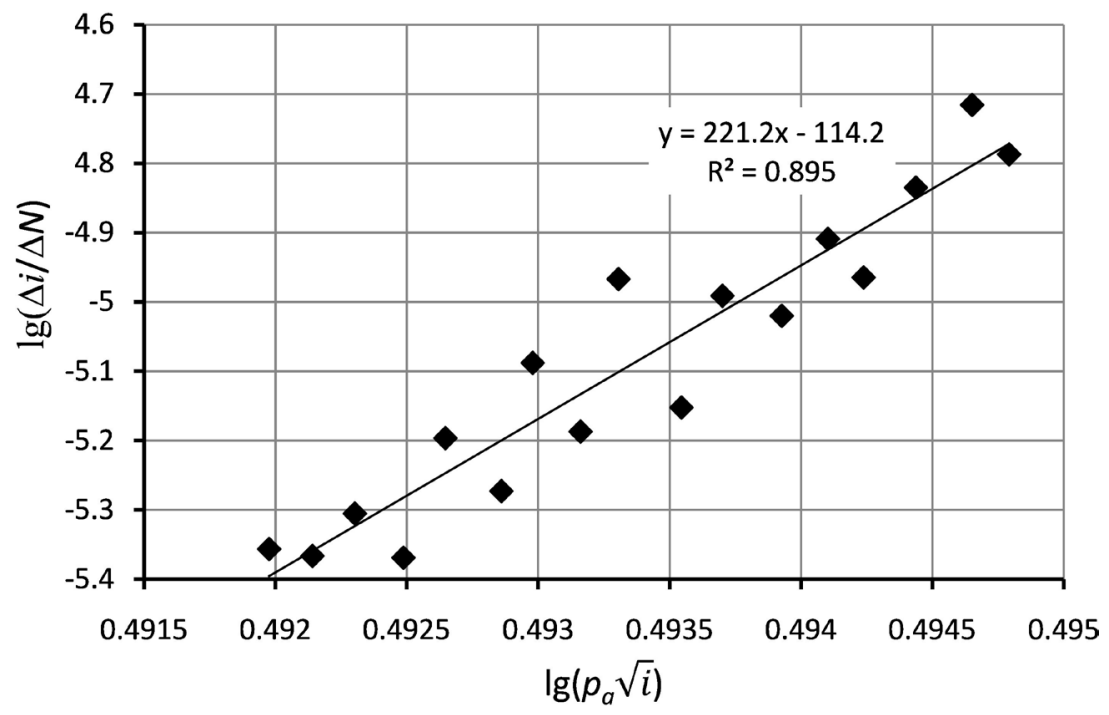

Figure 7. Graph of $i / F_{N}$ ratio as a function of $K_{\tau W}$ for a PTFE liner with a contact load of $280 \mathrm{~N}$ in logarithmic coordinates.

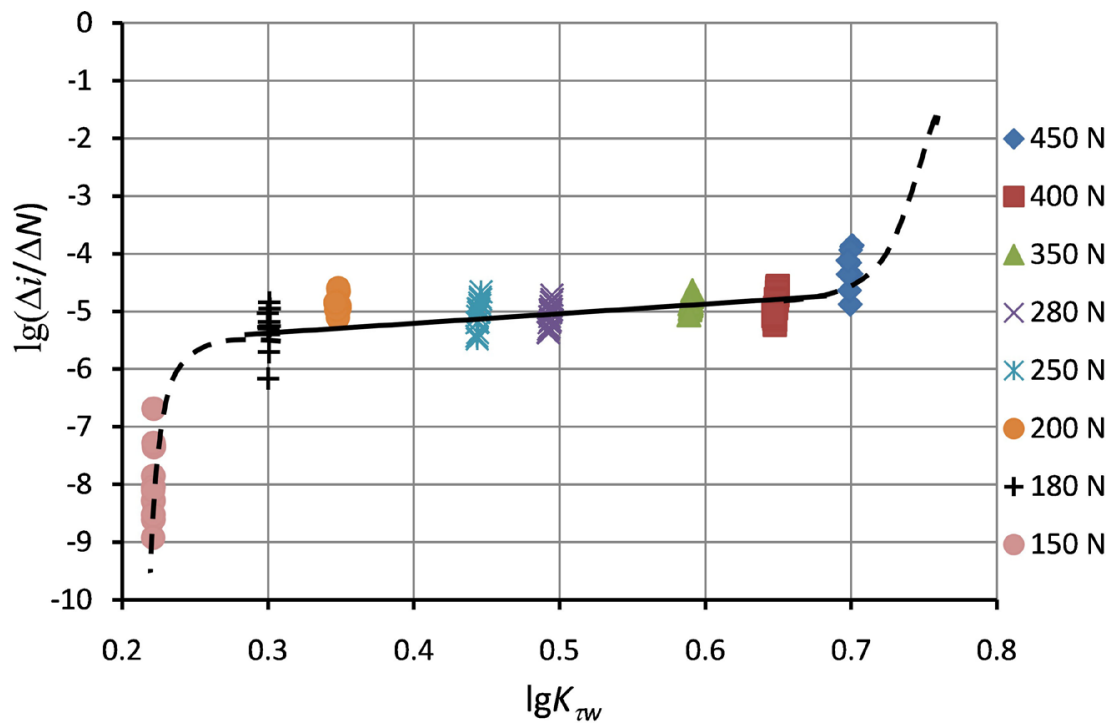

Figure 8. A generalized graph of the dependence of the $i / F_{N}$ ratio on the $K_{\mathrm{tw}}$ value for a PTFE liner with a contact load of 450, 400, 350, 280, 250, 200, 180 and $150 \mathrm{~N}$ in logarithmic coordinates. 
(Figure 6), then we can see that in the contact load range from 180 to $400 \mathrm{~N}$ we have a steady wear stage (it corresponds to section III in Figure 6), which satisfies the Equation (6) with the parameters $B^{*}=1.122 \times 10^{-6}, \beta^{*}=2.21$. Note that for a number of polymers $\beta=1.5 \cdots 3.0$ [9] [14] was set.

Obviously with contact loads smaller than $180 \mathrm{~N}$, we will have a stage of low wear rates (Figure 8 shows a curve going down), corresponding to section II of low growth rates of polymer cracks (Figure 6). With contact loads exceeding 400 - $450 \mathrm{~N}$, we obtain a stage of high wear rates (Figure 8 shows a dashed curve going up) corresponding to section IV of high crack growth rates (Figure 6). It should be noted that stage IV in Figure 8 is not obvious as no data point is plotted in the corresponding range. In the same way, the transition stage between I and II is not obvious.

\section{Conclusions}

Therefore, and according to the expressions stated in Equations (4)-(6), a clear analogy between the wear and the crack growth phenomena can be established, obtaining similar wear and crack growth rates, respectively. This is true for the friction pair studied as applied to the specified test conditions.

However, it is necessary to conduct additional experiments with other contact loads, other test conditions and other materials of a friction pair in order to assess the validity of the proposed approach to the description of wear kinetics during sliding friction. In addition, it is necessary to give a clear physical meaning to the parameters $B^{*}$ and $\beta^{*}$ of Equation (6).

\section{Conflicts of Interest}

The author declares no conflicts of interest regarding the publication of this paper.

\section{References}

[1] Kragelskii, I.V. (1959) Theory of Wear of Highly Elastic Materials. Soviet Rubber Technology, 5, 18-22.

[2] Kragelskii, I.V., Dobichin, M.N. and Kombalov, V.S. (1977) Basics of Calculations for Friction and Wear. Mechanical Engineering, Moscow.

[3] Sosnovskiy, L.A. (2005) Tribo-Fatigue. Wear-Fatigue Damage and Its Prediction Series: Foundations of Engineering Mechanics. Springer, Berlin. https://doi.org/10.1007/978-3-540-27027-0_2

[4] Harsha, A.P. and Tewari, U.S. (2003) Two-Body and Three-Body Abrasive Wear Behavior of Polyaryletherketone Composites. Polymer Tests, 22, 403-418. https://doi.org/10.1016/s0142-9418(02)00121-6

[5] Zhang, S.W. (2004) Tribology of Elastomers. In: Briscoe, B.J., Ed., Tribology and Interface Engineering, Series No. 47, 37-177.

[6] Jia, X. and Ling, R. (2007) Two-Body Free-Abrasive Wear of Polyethylene, Nylon1010, Epoxy and Polyurethane Coatings. Tribology International, 40, 1276-1283. https://doi.org/10.1016/j.triboint.2007.02.013

[7] Marchenko, E.A. (1990) Essentials of Friction Breakage for Metals Surface. National 
Defence Industry Press, Beijing, 1-8.

[8] Liu, J.J., Zhou, P.A., Sun, X.T., Liao, Q.C., et al. (1989) Adhesive Wear and Fatigue Wear of Materials. Machinery Industry Press, Beijing, 234-323.

[9] Martinez, F.J., Canales, M., Bielsa, J.M. and Jimenez M.A. (2010) Relationship between Wear Rate and Mechanical Fatigue in Sliding TPU-Metal Contacts. Wear, 268, 388-398. https://doi.org/10.1016/j.wear.2009.08.026

[10] Archard, J.F. (1953) Contact and Rubbing of Flat Surfaces. Journal of Applied Physics, 24, 981-988. https://doi.org/10.1063/1.1721448

[11] Rabinowicz, E. (1965) Friction and Wear of Materials. Wiley, New York.

[12] Champ, D.H., Southern, E. and Thomas, A.G. (1974) Fracture Mechanics Applied to Rubber Abrasion. American Chemical Society, Division of Organic Coatings Plastics, 34, 237.

[13] Cho, K. and Lee, D. (2000) Effect of Molecular Weight between Cross-Links on the Abrasion Behavior of Rubber by a Blade Abrader. Polymer, 41, 133-140. https://doi.org/10.1016/s0032-3861(99)00111-1

[14] McKeen, L. (1995) Fatigue and Tribological Properties of Plastics and Elastomers. Plastics Design Library, VI. 